SPhT-T05/152

DESY $05-186$

\title{
The GL(1|1) WZW-Model: From Supergeometry to Logarithmic CFT
}

\author{
Volker Schomerus and Hubert Saleur \\ DESY Theory Group, Notkestrasse 85 \\ D-22603 Hamburg, Germany. \\ Service de Physique Théorique, CEA Saclay, \\ F-91191 Gif-sur-Yvette, France; \\ Physics Department \\ University of Southern California \\ Los Angeles CA 90089-0484.
}

October, 2005

\begin{abstract}
We present a complete solution of the WZW model on the supergroup GL(1|1). Our analysis begins with a careful study of its minisuperspace limit ("harmonic analysis on the supergroup"). Its spectrum is shown to contain indecomposable representations. This is interpreted as a geometric signal for the appearance of logarithms in the correlators of the full field theory. We then discuss the representation theory of the $\operatorname{gl}(1 \mid 1)$ current algebra and propose an Ansatz for the state space of the WZW model. The latter is established through an explicit computation of the correlation function. We show in particular, that the 4-point functions of the theory factorize on the proposed set of states and that the model possesses an interesting spectral flow symmetry. The note concludes with some remarks on generalizations to other supergroups.
\end{abstract}

e-mail: volker.schomerus@desy.de, hubert.saleur@cea.fr 


\section{Introduction}

Throughout the last two decades, non-linear sigma models with super-manifold target spaces have emerged in a wide variety of systems and their study has become increasingly relevant for some of the challenging problems of modern physics, ranging from e.g. the quantum Hall effect to the famous AdS/CFT correspondence in string theory.

In condensed matter, super-manifold target spaces arise mostly in the study of geometrical problems such as percolation and polymers [1], or in non-interacting disordered systems [2, 3], where ill defined $n \rightarrow 0$ "replica" limits are handled instead by the introduction of fermionic degrees of freedom to, typically, cancel bosonic loops. The transition between plateaux in the integer quantum Hall effect is thus believed to be related to the sigma model $\mathrm{U}(1,1 \mid 2) / \mathrm{U}(1 \mid 1) \times \mathrm{U}(1 \mid 1)$ at $\theta=\pi$, a conformal field theory which has not yet been understood, despite decades of work (for a recent attempt, see 4]). Slightly more progress has been made for geometrical loop models, leading to partial solutions of sigma models on $\mathrm{U}(\mathrm{n}+\mathrm{m} \mid \mathrm{n}) / \mathrm{U}(1) \times \mathrm{U}(\mathrm{n}+\mathrm{m}-1 \mid \mathrm{n})$ (super projective spaces) and $\operatorname{Osp}(2 \mathrm{n}+\mathrm{m} \mid 2 \mathrm{n}) / \operatorname{Osp}(2 \mathrm{n}+\mathrm{m}-1 \mid 2 \mathrm{n})$ (superspheres) [5] .

In string theory, super-manifold target spaces received brief attention about then years ago when they were argued to arise as mirrors of rigid Calabi-Yau (CY) manifolds, i.e. of CY spaces without complex moduli. According to the usual rules, the mirror image of such spaces has no Kähler moduli and hence it cannot be a usual CY manifold. In [6] Sethi argued that the dual of a rigid CY is instead given by a CY super-manifold. The proposal was further investigated in a small number of subsequent publications (see e.g. [7, 8] and references therein), but it did not trigger much interest in sigma models with super-target spaces. Mirror symmetry (or T-duality) involving non-commutative geometries, of which super-manifolds are the simplest examples, has also been discussed recently in [9, 10, 11].

Presumably more important, however, is the role that super-group and super-coset targets play for the description of strings in Anti-deSitter spaces. Using the GreenSchwarz formalism, a link was first established by Metsaev and Tseytlin [12. Shortly

after, Berkovits, Vafa and Witten explained [13] how string theory on $A d S_{3} \times S^{3}$ could be quantized if it was possible to construct conformal quantum field theories with a PSL $(2 \mid 2)$ target space. Such models were investigated in an interesting paper by Bershadsky [14] in which some of the peculiar features of super-target spaces surfaced. For further string 
motivated research in this direction see e.g. [15, 16, 17, and for more condensed matter oriented work see [19, 20, 21, 22]).

In most circumstances, the models of interest are believed to be more complicated than WZW models on supergroups. In the case of the integer quantum Hall effect for instance, it has become clear over the years that the sigma model at $\theta=\pi$ flows to a theory which does not exhibit the full current algebra symmetry [5] (presumably because of the appearance of logarithmic terms in the OPEs of the currents). Nevertheless, even the WZW models on supergroups are far from being understood. This is largely due to technical reasons (indecomposability of operator products and appearance of logarithms in correlation functions, continuous modular transforms of the irreducible characters [23]...), combined with a lack of "physical intuition".

Our aim in this note is to initiate a systematic study of WZW models on supergroups by relying more heavily on geometric concepts. We shall, in particular, gain a better understanding of logarithmic features by relating them to super-geometry. Logarithmic conformal field theories have been studied for a bit more than a decade now (see [24, 25] for some early contributions). Even though only a few examples have been constructed in full detail [26], their importance, in particular for disordered critical points, is widely appreciated (see e.g. [27, 28, 5, 29] and references therein). By definition, the operator product expansions in a logarithmic conformal field theory contain a logarithmic dependence on the separation between the fields. In the simplest cases, these may look e.g. as follows,

$$
\Phi(x, \bar{x}) \Phi(0,0) \sim \frac{1}{|x|^{2 \Delta_{\Phi}-2 h_{C}}}\left(\log |x|^{2} C(0,0)+D(0,0)\right)+\ldots
$$

We conclude that the chiral generators $L_{0}$ and $\bar{L}_{0}$ of dilations in the world-sheet coordinate $x$ act according to

$$
L_{0}|D\rangle=h_{C}|D\rangle+|C\rangle \quad, \quad L_{0}|C\rangle=h_{C}|C\rangle
$$

Here, $|C\rangle,|D\rangle$ denote the states that are associated with the fields $C, D$ and similar relations hold for $\bar{L}_{0}$. Consequently, $L_{0}$ and $\bar{L}_{0}$ cease to be diagonalizable. This feature is common to all logarithmic conformal field theories and it is rather easy to diagnose. Many more details and references may be found in recent review articles [30, 31].

Our strategy here is to approach the analysis of the WZW model through the harmonic analysis on the supergroup GL(1|1) and to show that the minisuperspace analogues of $L_{0}$ 
and $\bar{L}_{0}$, i.e. the quadratic Casimir elements in the left and right regular representations, are non-diagonalizable. This leaves the full field theoretic model no other chance but to be logarithmic. The harmonic analysis on the supergroup GL(1|1) is the main subject of the next section. In section 3 we will suggest an expression for the state space of the full field theory. Our proposal is motivated in parts by the experience with the minisuperspace theory combined with some results from the representation theory of the gl(1|1) current algebra. It is established later through a full construction of the theory, including all its correlators. Our solution is based on free field computations involving a $c=2$ linear dilaton in the bosonic sector and an (anti-)chiral bc-system with central charge $c=-2$ for the fermionic part. All 3-point functions of the model are constructed and studied in section 5. There we shall also show that the theory possesses an interesting spectral flow symmetry. In section 6 , finally, we determine 4-point functions of our model an show that they factorize on the proposed set of states. We conclude with a few remarks on generalizations to other supergroups and with an outlook on further open problems.

\section{The minisuperspace analysis}

The following section is devoted to the "particle limit" of the GL(1|1) WZW model . In more physical terms, one can imagine putting the WZW model on a cylinder with periodic space and infinite (imaginary) time, and restricting to zero modes, ie to field configurations that are independent of the space variable. Their dynamics is the one of a particle with

phase space the target space of the WZW model. Thus, in more mathematical terms we shall be concerned with the harmonic analysis on GL(1|1). Such harmonic analysis has been quite successful in the study of WZW models on non-compact bosonic target spaces such as the $\mathrm{SL}(2, \mathrm{C}) / \mathrm{SU}(2)$ model (see e.g. [32]).

We will require a bit of background from the representation theory of the Lie superalgebra gl(1|1). In particular we shall introduce its typical representations (long multiplets) and show how they generate certain indecomposable composites of atypical representations (short multiplets) through tensor products. We then construct the space of functions on the supergroup along with the left and right regular action of $\mathrm{gl}(1 \mid 1)$. The regular representation is explicitly decomposed into its building blocks and it is shown that indecomposable (but not irreducible) representations emerge in the spectrum. Part of the results we discuss here were first derived in 33 . 


\subsection{The Lie superalgebra $\operatorname{gl}(1 \mid 1)$ and its representations}

The Lie superalgebra $\mathfrak{g}=\operatorname{gl}(1 \mid 1)$ is generated by two bosonic elements $E, N$ and two fermionic generators $\Psi^{ \pm}$such that $E$ is central and the other generators obey

$$
\left[N, \Psi^{ \pm}\right]= \pm \Psi^{ \pm} \quad \text { and } \quad\left\{\Psi^{-}, \Psi^{+}\right\}=E .
$$

Let us also fix the following Casimir element $C$ for gl(1|1)

$$
C=(2 N-1) E+2 \Psi^{-} \Psi^{+} .
$$

The choice of $C$ is not unique since we could add any function of the central element $E$. Our prescription is motivated by the form of the Virasoro element in the field theory (see 24] and below).

There are five different classes of representations that shall play some role in the following. To begin with, we list the irreducible representations which fall into the different series. There is one series of 2-dimensional representations $\langle e, n\rangle$ which is labeled by pairs $e, n$ with $e \neq 0$ and $n \in \mathbb{R}$. In these representations, the generators take the form $E=e \mathbf{1}_{2}$ and

$$
N=\left(\begin{array}{cc}
n-1 & 0 \\
0 & n
\end{array}\right), \quad \Psi^{+}=\left(\begin{array}{ll}
0 & 0 \\
e & 0
\end{array}\right), \quad \Psi^{-}=\left(\begin{array}{ll}
0 & 1 \\
0 & 0
\end{array}\right) .
$$

These representations are the typical representations (long multiplets) of $\mathfrak{g}=\operatorname{gl}(1 \mid 1)$. In addition, there is one series of atypical representations $\langle n\rangle$ (short multiplets). These are 1-dimensional and parametrized by the value $n \in \mathbb{R}$ of $N$. All other generators vanish.

For the typical representations we assumed that the parameter $e$ does not vanish. But it is still interesting to explore what happens when we set $e=0$. The above matrices certainly continue to provide a representation of gl(1|1) only that this is no longer irreducible. In fact, we observe that the basis vector $|0\rangle=(1,0)^{T}$ generates a 1-dimensional invariant subspace of the corresponding 2-dimensional representation space. But one should not conclude that there exits an invariant complement. In fact, it is impossible to decouple the vector $|1\rangle=(0,1)^{T}$ from the representation since $\Psi^{-}|1\rangle=|0\rangle$, independently of the choice of the parameter $e$. The representation $\langle 0, n\rangle$ is therefore indecomposable but it is not irreducible. We can think of $\langle 0, n\rangle$ as being built up from two atypical constituents, namely from the representations $\langle n\rangle$ and $\langle n-1\rangle$. To visualize the internal structure of $\langle 0, n\rangle$, we may employ the following diagram,

$$
\langle 0, n\rangle:\langle n-1\rangle \longleftarrow\langle n\rangle .
$$


Later we shall see much more complicated composites of atypical representations. It is therefore useful to become familiar with diagrammatic presentations of indecomposables.

In the representations $\langle e, n\rangle$, the fermionic generators appear on a somewhat different footing since $\Psi^{+}$depends on the parameter $e$ while $\Psi^{-}$does not. There exists another family of 2-dimensional representations $\langle\overline{e, n}\rangle$, however, in which the roles of $\Psi^{-}$and $\Psi^{+}$ are interchanged,

$$
N=\left(\begin{array}{cc}
n & 0 \\
0 & n-1
\end{array}\right), \quad \Psi^{+}=\left(\begin{array}{ll}
0 & 1 \\
0 & 0
\end{array}\right), \quad \Psi^{-}=\left(\begin{array}{ll}
0 & 0 \\
e & 0
\end{array}\right) .
$$

As long as $e \neq 0$ the representations $\langle e, n\rangle$ and $\langle\overline{e, n}\rangle$ are equivalent. In fact, the isomorphism between the two representations may be implemented by conjugation with the matrices $W_{e}=e \sigma^{+}+\sigma^{-}$where $\sigma^{ \pm}$are the usual Pauli matrices. This isomorphism does not survive the limit $e \rightarrow 0$ and hence the representations $\langle 0, n\rangle$ and $\langle\overline{0, n}\rangle$ are inequivalent. $\langle\overline{0, n}\rangle$ is also an indecomposable representation that is built up from the same atypical constituents as $\langle 0, n\rangle$, but this time the non-vanishing generator $\Psi^{+}$maps us from $\langle n\rangle$ to $\langle n-1\rangle$, i.e.

$$
\langle\overline{0, n}\rangle:\langle n-1\rangle \longrightarrow\langle n\rangle
$$

Below, the representations $\langle 0, n\rangle$ and $\langle\overline{0, n}\rangle$ will eventually enter as limits of typical representations.

Having seen all the irreducible representations $\langle e, n\rangle$ and $\langle n\rangle$ of $\operatorname{gl}(1 \mid 1)$ along with their limits as $e$ goes to zero, our next task is to compute tensor products of typical representations $\left\langle e_{1}, n_{2}\right\rangle$ and $\left\langle e_{2}, n_{2}\right\rangle$. As long as $e_{1}+e_{2} \neq 0$, the tensor product is easily seen to decompose into a sum of two typicals,

$$
\left\langle e_{1}, n_{2}\right\rangle \otimes\left\langle e_{2}, n_{2}\right\rangle=\left\langle e_{1}+e_{2}, n_{1}+n_{2}-1\right\rangle \oplus\left\langle e_{1}+e_{2}, n_{1}+n_{2}\right\rangle
$$

But when $e_{1}+e_{2}=0$ we obtain a 4 -dimensional representation that cannot be decomposed into a direct sum of smaller subrepresentations. The representation matrices of these 4dimensional indecomposables $\mathcal{P}_{n}$ read as follows

$N=\left(\begin{array}{cccc}n-1 & 0 & 0 & 0 \\ 0 & n & 0 & 0 \\ 0 & 0 & n & 0 \\ 0 & 0 & 0 & n+1\end{array}\right) \quad, \quad \Psi^{+}=\left(\begin{array}{cccc}0 & 0 & 0 & 0 \\ -1 & 0 & 0 & 0 \\ 1 & 0 & 0 & 0 \\ 0 & 1 & 1 & 0\end{array}\right), \quad \Psi^{-}=\left(\begin{array}{cccc}0 & 1 & 1 & 0 \\ 0 & 0 & 0 & 1 \\ 0 & 0 & 0 & -1 \\ 0 & 0 & 0 & 0\end{array}\right)$.

As we have seen before, it is useful to picture the structure of indecomposables. The form of $N$ tells us that $\mathcal{P}_{n}$ is composed from the atypical irreducibles $\langle n-1\rangle, 2\langle n\rangle,\langle n+1\rangle$. 
The action of $\Psi^{ \pm}$relates these four representations as follows

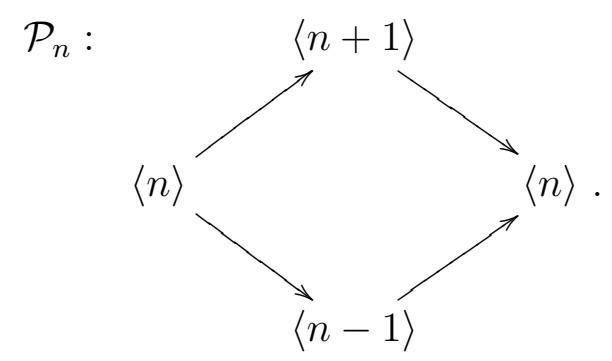

There are a few remarks we would like to make at this point. The first one concerns the form of the Casimir element $C$ in the representations $\mathcal{P}_{n}$. It is straightforward to see that $C$ maps the subspace $\langle n\rangle$ on the left onto the $\langle n\rangle$ on the right of the above diagram and that it is zero otherwise. This means that $C$ cannot be diagonalized in $\mathcal{P}_{n}$. We shall return to this observation later on.

It is also obvious from the diagrammatic representation that $\mathcal{P}_{n}$ contains the indecomposables $\langle 0, n\rangle$ and $\langle\overline{0, n+1}\rangle$ as subrepresentations. In this sense, the latter are extendable into a larger indecomposable. For the representation $\mathcal{P}_{n}$ the situation is quite different: it may be shown (and is intuitively clear) that $\mathcal{P}_{n}$ is maximal in the sense that it can never appear as a subrepresentation of a larger indecomposable. In the mathematics literature, such representations are known as projective. Since the projective representation $\mathcal{P}_{n}$ contains the irreducible $\langle n\rangle$ as a true subrepresentation, one also calls $\mathcal{P}_{n}$ the projective cover of $\langle n\rangle$.

The typical representations $\langle e, n\rangle, e \neq 0$, along with the indecomposables $\mathcal{P}_{n}$ exhaust the set of finite dimensional projectives of $\mathrm{gl}(1 \mid 1)$. What will be particularly important for us is the fact that projective representations are known to close under tensor products. In particular, tensor products of the representations $\mathcal{P}_{n}$ do not generate any new types of representations. This is not to say that there are not any others. In fact, there is a large family of indecomposables ("zigzag modules") with arbitrarily large dimension (see e.g. 34 for a complete list and a computation of their tensor products). Our following analysis will shortly confirm the standard mathematical result that only projectives emerge from the harmonic analysis on the supergroup and hence these are the only ones that will play a major role below. 


\subsection{Harmonic analysis on the supergroup GL(1|1)}

Our aim now is to study the space of functions on the corresponding supergroup and to analyse the various actions of the Lie superalgebra gl(1|1). Before we get into the details, however, let us briefly recall the situation in the case of compact groups which is covered by Peter-Weyl theory. The latter describes how the space $L_{2}(G)$ of square integrable functions on a compact group decomposes under the right regular action. It asserts that the infinite dimensional representation space $L_{2}(G)$ decomposes into a direct sum of irreducibles $H_{J}$ of $G$ and that each irreducible appears with a multiplicity that is given by the dimension $d_{J}$ of $H_{J}$, i.e.

$$
L_{2}(\mathrm{G}) \cong \sum_{J} H_{J} \otimes H_{J}^{R}
$$

Here, the first factor $H_{J}$ in each summand is the multiplicity space. The generators of the right regular representation act exclusively in the second tensor factor which is why we marked it with the superscript $R$.

We can actually be even more precise and construct each summand in the above decomposition rather explicitly. To this end we note that the Hilbert space $L_{2}(G)$ possesses a basis which is formed by matrix elements of irreducible representations of $G$. Any irreducible representation $H_{J}$ contributes $d_{J}^{2}$ matrix elements to the basis. These span the subspaces $H_{J} \otimes H_{J}^{R}$ in the above decomposition of $L_{2}(G)$.

Obviously, there exists a second action of $G$ on $L_{2}(G)$ by left multiplication. It promotes the multiplicity spaces $H_{J}$ into representation spaces of $G$, i.e.

$$
L_{2}(G) \cong \sum_{J} H_{J}^{L} \otimes H_{J}^{R}
$$

The structure of this decomposition under the combined left and right action is somewhat reminiscent of the famous holomorphic factorization in WZW models.

In the following discussion of functions on the supergroup, we would like to remain very explicit. Therefore, we introduce the so-called Gauss coordinates $x, y, \eta_{ \pm}$in which elements of the supergroup read

$$
U=U\left(x, y, \eta_{ \pm}\right)=e^{i \eta_{+} \Psi^{+}} e^{i x E+i y N} e^{i \eta_{-} \Psi^{-}} .
$$

It is not hard to work out the form of the invariant measure in these coordinates. The result is

$$
d \mu=e^{-i y} d x d y d \eta_{-} d \eta_{+}
$$


Similarly, one can determine the form of the left and right invariant vector fields. Again we only quote the results of a straightforward computation. For the left invariant vector fields one finds

$$
L_{E}=i \partial_{x}, \quad L_{N}=i \partial_{y}-\eta_{+} \partial_{+}, \quad L_{+}=-i \partial_{+}, \quad L_{-}=i e^{i y} \partial_{-}-\eta_{+} \partial_{x}
$$

Here, the symbols $\partial_{ \pm}$stand for derivatives with respect to $\eta_{ \pm}$. Right invariant vector fields possess the form

$$
R_{E}=-i \partial_{x}, R_{N}=-i \partial_{y}+\eta_{-} \partial_{-}, R_{-}=-i \partial_{-}, R_{+}=i e^{i y} \partial_{+}+\eta_{-} \partial_{x}
$$

The reader is invited to check that these two sets of generators satisfy the relations of the Lie superalgebra $\mathrm{gl}(1 \mid 1)$ and that they (anti-)commute among each other.

After this preparation, we would like to analyze the space of square integrable functions on the supergroup. By definition, these are objects $f$ of the form

$$
f\left(x, y, \eta_{ \pm}\right)=f_{0}(x, y)+f_{+}(x, y) \eta_{+}+f_{-}(x, y) \eta_{-}+f_{2}(x, y) \eta_{-} \eta_{+}
$$

with any set of square integrable functions $f_{\nu}$ on $\mathbb{R}^{2}$. This space is spanned by the following basis $^{1}$

$$
e_{0}(k, l)=e^{i k x+i l y}, e_{ \pm}(k, l)=e_{0}(k, l) \eta_{ \pm}, e_{2}(k, l)=e_{0}(k, l) \eta_{-} \eta_{+} .
$$

The space of square integrable functions carries two (anti-)commuting actions of the Lie superalgebra gl(1|1) which are generated by the left- and right invariant vector fields. Our aim is to understand in detail the structure of these representations.

Proposition 1: (Right regular action) With respect to the right regular action, the space of square integrable functions on the supergroup decomposes according to

$$
L_{2}(\mathrm{GL}(1 \mid 1))=\int_{e \neq 0} \operatorname{dedn}\left(H_{\langle e, n\rangle}^{\prime} \oplus H_{\langle e, n\rangle}^{R}\right) \oplus \int d n \mathcal{P}_{n}
$$

Here $H_{\langle e, n\rangle}^{R}$ denotes the graded representation space of the typical representation $\langle e, n\rangle$ and $H_{\langle e, n\rangle}^{\prime}$ is the same vector space with shifted $\mathbb{Z}_{2}$ grading.

Let us make a few remarks about this result before we explain its derivation. The two integrals in our decomposition formula correspond to an integration over the space of typical and atypical representations, respectively. As in the case of ordinary groups, typical

\footnotetext{
${ }^{1}$ The elements of this basis are $\delta$-function normalizable since we are dealing with a non-compact group.
} 
representations appear with a multiplicity given by their dimension, i.e. by $d_{\langle e, n\rangle}=2$ in our special case. For atypical representations, the story is more complicated. In general, they do not appear themselves but are replaced by their projective covers. Their multiplicity, on the other hand, is obtained from the dimension of the atypical representation, i.e. by $d_{\langle n\rangle}=1$ in our special case. ${ }^{2}$ Our decomposition formula is thus in full agreement with the general result in 33 . Note that the structure of the sector which comes with the atypical representations does not possess the usual form that is encoded in the Peter-Weyl theorem (see above).

We also mention is passing that the Casimir element $C$ is non-diagonalizable in the right regular representation since the latter contains the projective covers and, according to our earlier discussion, $C$ cannot be diagonalized in $\mathcal{P}_{n}$. In the full field theory, the Casimir elements lifts to the Virasoro zero mode $L_{0}$ so that our simple corollary on the structure of $C$ in the right regular representation will eventually have direct and far reaching implications for the WZW model.

Our result on the decomposition of the right regular representation is rather easy to obtain and we can even find explicit formulas for the basis vectors of all the summands. In order to do so, we shall have a brief look at the space of functions that appear as matrix elements of the supergroup in the typical representations $\langle e, n\rangle$,

$$
\varphi_{\langle e, n\rangle}=\left(\begin{array}{cc}
e^{i e x+i(n-1) y} & i \eta_{-} e^{i e x+i(n-1) y} \\
i e \eta_{+} e^{i e x+i(n-1) y} & e \eta_{-} \eta_{+} e^{i e x+i(n-1) y}+e^{i e x+i n y}
\end{array}\right) .
$$

The functions in the first row form a basis of the summand $H_{\langle e, n\rangle}$, whereas the functions in the second row span the space $H_{\langle e, n\rangle}^{\prime}$,

$$
\begin{aligned}
& H_{\langle e, n\rangle}=\operatorname{span}\left(e_{0}(e, n-1), e_{-}(e, n-1)\right) \\
& H_{\langle e, n\rangle}^{\prime}=\operatorname{span}\left(e_{+}(e, n-1), e e_{2}(e, n-1)+e_{0}(e, n)\right) .
\end{aligned}
$$

It is obvious that the matrix elements of the typical representations provide a basis for eigenfunctions of $R_{E}$ with eigenvalue $e \neq 0$.

What we are missing is an analysis of the space of functions with $e=0$. The space of these functions is spanned by $e_{\nu}(0, l)$ and it is easily seen to decompose into a sum of 4-dimensional indecomposables $\mathcal{P}_{n}$,

$$
\mathcal{P}_{n}=\operatorname{span}\left(e_{0}(0, n), e_{+}(0, n-1), e_{-}(0, n), e_{2}(0, n-1)\right) .
$$

\footnotetext{
${ }^{2}$ For some Lie superalgebras, there can be representations for which the multiplicity is only half of this value.
} 
One may check by direct computation that $R_{ \pm} e_{2}(0, n-1)= \pm i e_{\mp}(0, n-1 / 2 \pm 1 / 2)$ and similarly that $R_{ \pm} e_{ \pm}(0, n-1 / 2 \mp 1 / 2)=-i e_{0}(0, n)$. Hence, we recover the structure of the projective cover $\mathcal{P}_{n}$.

The functions on our supergroup carry another (anti-)commuting action of the Lie superalgebra $\mathfrak{g}$ by left derivations. There is a corresponding decomposition which is certainly identical to the decomposition in proposition 1. A more interesting problem is to decompose the space of functions with respect to the graded product $\mathfrak{g} \otimes \mathfrak{g}$ in which the first factors acts through the left regular action while for the second factor we use the right regular action. The associated decomposition is provided by the following proposition.

Proposition 2: (Left-right regular action) With respect to the left-right regular action of $\mathfrak{g} \otimes \mathfrak{g}$, the space of functions on the supergroup decomposes according to

$$
L_{2}(\mathrm{GL}(1 \mid 1))=\int_{e \neq 0} \operatorname{dedn} H_{\langle-e,-n+1\rangle}^{L} \otimes H_{\langle e, n\rangle}^{R} \oplus \int_{0}^{1} d q \mathcal{J}_{q} .
$$

Here $\mathcal{J}_{q}, q \in[0,1[$, denotes a a family of infinite dimensional indecomposable representation of $\mathfrak{g} \otimes \mathfrak{g}$. When restricted to either left or right regular action, the latter decompose according to

$$
\left(\mathcal{J}_{q}\right)_{\mathfrak{g}^{R}} \sim\left(\mathcal{J}_{-q}\right)_{\mathfrak{g}^{L}}=\bigoplus_{a \in \mathbb{Z}} \mathcal{P}_{q+a} \quad \text { for all } \quad q \in[0,1[.
$$

The first term in the decomposition formula follows from proposition 1 as in the case of Lie algebras. Our second term involves unusual infinite dimensional representations which cannot be further decomposed. They appear as follows. We have displayed an explicit basis for the 4-dimensional spaces $\mathcal{P}_{n}$ of the right regular action in eq. (2.5): from this it is easy to see that

$$
L_{-}: \mathcal{P}_{n} \longrightarrow \mathcal{P}_{n+1} \quad, \quad L_{+}: \mathcal{P}_{n} \longrightarrow \mathcal{P}_{n-1}
$$

i.e. that the invariant subspaces $\mathcal{P}_{n}$ of the right regular action are mapped into each other by the left regular action and vice versa, thus "linking" the projectives for left and right actions into a big block. In terms of its decomposition series, the structure of $\mathcal{J}_{q}$ is given 
by

$$
\begin{aligned}
& \mathcal{J}_{q}: \quad \bigoplus_{a \in \mathbb{Z}}\langle q+a\rangle \otimes\langle-q-a\rangle \\
& \longrightarrow \bigoplus_{a \in \mathbb{Z}}\langle q+a+1\rangle \otimes\langle-q-a\rangle \oplus \bigoplus_{a \in \mathbb{Z}}\langle q+a\rangle \otimes\langle-q-a-1\rangle \\
& \quad \longrightarrow \bigoplus_{a \in \mathbb{Z}}\langle q+a\rangle \otimes\langle-q-a\rangle .
\end{aligned}
$$

Note that $\langle n\rangle \otimes\langle m\rangle$ are atypical 1-dimensional representations of $\mathfrak{g} \otimes \mathfrak{g}$. The rightmost term in this filtration of $\mathcal{J}_{q}$ denotes the so-called socle, i.e. the largest semi-simple submodule. The leftmost term is the head of $\mathcal{J}_{q}$. It is the largest semi-simple representation that arises as a quotient of $J_{q}$. The term in the middle, finally, is the head of the radical.

\subsection{Correlation functions in minisuperspace}

In the minisuperspace theory, fields are represented through functions $\phi$ on the supergroup and their correlators are computed by integration with the invariant measure, i.e.

$$
\left\langle\prod_{\nu=1}^{m} \phi_{\nu}\right\rangle=\int d \mu\left(x, y, \eta_{ \pm}\right) \prod_{\nu=1}^{m} \phi_{\nu}\left(x, y, \eta_{ \pm}\right)
$$

Here we shall be mostly concerned with the correlators involving matrix elements of the objects $\varphi_{\langle e, n\rangle}$.

In order to prepare for our analysis of correlators of typical fields we need to introduce a bit of notation. As before, we shall denote the eigenstates of $N$ in typical representations by $|0\rangle$ and $|1\rangle$. Our choice is such that $\Psi^{-}|0\rangle=0$. States of an m-fold tensor product can be thought of as states in a spin chain of length $m$. A basis in this space is given by $\left|\sigma_{1} \ldots \sigma_{m}\right\rangle$ with $\sigma_{i}=0,1$. In this vector space we shall introduce a set of linear maps $E$ by

$$
E_{\sigma_{1}^{\prime} \ldots \sigma_{m}^{\prime}}^{\sigma_{1} \ldots \sigma_{m}}=\left|\sigma_{1} \ldots \sigma_{m}\right\rangle\left\langle\sigma_{1}^{\prime} \ldots \sigma_{m}^{\prime}\right|
$$

These are the elementary matrices of the state space of our spin chain. They will appear later in our formulas for the correlation functions of primary fields that are associated with typical representations.

When we evaluate $m$-point functions of our matrix valued functions $\varphi_{\langle e, n\rangle}$, we can express the answer in terms of the elementary matrices $E$ for a spin chain of length $m$. 
Note that only elementary matrices can arise that preserve the number $p$ of spins that are flipped to the position $\sigma=1$. We can also observe that an elementary matrix with $p$ flipped spins comes multiplied by a delta function $\delta\left(\sum_{\nu=1}^{m} n_{\nu}-m+p-2\right)$, i.e. an m-point function possesses the general form ${ }^{3}$

$$
\left\langle\prod_{\nu=1}^{m} \varphi_{\left\langle e_{\nu}, n_{\nu}\right\rangle}\right\rangle=\delta\left(\sum_{\nu=1}^{m} e_{\nu}\right) \sum_{p=1}^{m-1} G_{p}^{(m)} \delta\left(\sum_{\nu=1}^{m} n_{\nu}-m+p-2\right)
$$

where $G_{p}^{(m)}$ are linear combinations of the elementary matrices for a spin chain of length $m$ with $p$ spins in the $\sigma=1$ position. We shall find the same structure for the correlators in the field theory later on. There is one more rule, however, that is specific to the particle limit: an elementary matrix can only contribute to the invariant tensor $G_{p}^{(m)}$ if it shifts at most one spin along the chain. For $m \leq 3$ this conditions is trivially satisfied, but starting from 4-point functions, some number preserving elementary matrices are no longer admitted in the particle limit. We shall see below that this last condition may be violated for the full field theory.

\section{Representation theory of current algebra}

Based on the experience from the previous section we would now like to present a similar analysis of the representation theory of the affine algebra. This will ultimately lead us to a conjecture on the state space of the GL(1|1) WZW model. Our proposal will follow closely the outcome of the harmonic analysis on the supergroup. The only new ingredient enters through an additional spectral flow symmetry of the affine algebra.

\subsection{The gl(1|1) current algebra}

Let us begin by listing a few results on the affine algebra and its representation theory. The gl(1|1) current algebra is generated by the modes of two bosonic currents $N(z), E(z)$ and two fermionic currents $\Psi^{ \pm}(z)$. Their commutation relations read

$$
\begin{gathered}
{\left[E_{n}, N_{m}\right]=k m \delta_{n+m} \quad, \quad\left[N_{n}, \Psi_{m}^{ \pm}\right]= \pm \Psi_{n+m}^{ \pm}} \\
\left\{\Psi_{n}^{-}, \Psi_{m}^{+}\right\}=E_{n+m}+k m \delta_{n+m} .
\end{gathered}
$$

\footnotetext{
${ }^{3}$ The term with $p=m$ vanishes due to conservation of the $e$-charge
} 
All other (anti-)commutators vanish. This algebra admits an interesting family of spectral flow automorphism $\gamma_{m}$ which acts on generators according to

$$
\gamma_{m}\left(E_{n}\right)=E_{n}+m k \delta_{n} \quad, \quad \gamma_{m}\left(\Psi_{n}^{ \pm}\right)=\Psi_{n \pm m}^{ \pm}
$$

and leaves $N_{n}$ invariant. We shall see later that for integer $m$ these automorphisms provide a symmetry of the GL(1|1) WZW model.

In [24] it was shown that the the Virasoro element $L_{0}$ of the gl(1|1) model possesses the following form,

$$
\begin{aligned}
L_{0}= & \frac{1}{2 k}\left(2 N_{0} E_{0}-E_{0}+2 \Psi_{0}^{-} \Psi_{0}^{+}+\frac{1}{k} E_{0}^{2}\right) \\
& +\frac{1}{k} \sum_{m \geq 0}\left(E_{-m} N_{m}+N_{-m} E_{m}+\Psi_{-m}^{-} \Psi_{m}^{+}-\Psi_{-m}^{+} \Psi_{m}^{-}+\frac{1}{k} E_{-m} E_{m}\right)
\end{aligned}
$$

Under the action of the spectral flow automorphism, $L_{0}$ behaves according to

$$
\gamma_{m}\left(L_{0}\right)=L_{0}+m\left(N_{0}-1\right)
$$

This very simple behavior of $L_{0}$ plays an important role in determining the action of the spectral flow on representations of the current algebra.

\subsection{Representations of the $\operatorname{gl}(1 \mid 1)$ current algebra}

In the following we shall denote the Verma module over the typical representation $\langle e, n\rangle$ by $\mathcal{V}_{\langle e, n\rangle}$. As long as $e$ is not an integer multiple of the level $k$, the Verma modules are irreducible. But when $e=k m$, the story is a bit more interesting.

Lemma: The Verma module that is built on the typical representations $\langle m k, n\rangle, m \neq 0$, contains a singular vector on the $m^{\text {th }}$ level. Explicitly, it is given by

$$
|k m, n \pm 1\rangle_{\langle m k, n\rangle}=\prod_{p=1}^{|m-1|} \Psi_{p}^{\mp} \prod_{p=1}^{|m|} \Psi_{-p}^{ \pm}|m k, n\rangle \quad \text { for } \quad 0< \pm m
$$

where $|m k, n\rangle$ denotes the ground state of the Verma module $\mathcal{V}_{\langle m k, n\rangle}$.

ProOF: Without loss of generality, let us restrict to $m>0$. In order to prove our statement we shall begin with the following simple fact,

$$
\Psi_{q}^{-} \prod_{p=1}^{m} \Psi_{-p}^{+}|m k, n\rangle=0 \quad \text { for all } \quad q \geq m
$$


For $q>m$, the formula would hold regardless of the ground state we use. Only the case $q=m$ is slightly more subtle and it uses that $E_{0}=m k$. With this insight in mind it is then straightforward to establish the Lemma.

If we divide the Verma module $\mathcal{V}_{\langle m k, n\rangle}$ by the invariant subspace that is generated from its singular vector (3.9) we end up with an irreducible representation of the current algebra. We shall denote the latter by $\mathcal{H}_{\langle m k, n\rangle}$. One may also show that the invariant subspace which is built on the vector (3.9) is irreducible and isomorphic to $\mathcal{H}_{\langle m k, n \pm 1\rangle}$. Hence, the structure of the Verma module $\mathcal{V}_{\langle e, n\rangle}$ is encoded in the following diagram

$$
\mathcal{V}_{\langle m k, n\rangle}: \quad \mathcal{H}_{\langle m k, n\rangle} \longrightarrow \mathcal{H}_{\langle m k, n \pm 1\rangle} \quad \text { for } \quad 0< \pm m
$$

For negative $m$, this resembles the structure of the module $\langle\overline{0, n}\rangle$. And indeed, one can easily see that the Verma module $\mathcal{V}_{\langle k m, n\rangle}$ for $m<0$ is the spectral flow image of the Verma module $\mathcal{V}_{\langle\overline{0, n}\rangle}$. Similarly, for positive $m$, the spectral flow takes us from the Verma module $\mathcal{V}_{\langle 0, n+1\rangle}$ to the Verma module $\mathcal{V}_{\langle k m, n\rangle}$.

In order to discuss the action of the spectral flow on the Verma modules over the projective covers $\mathcal{P}_{n}$, we need to enlarge the class of affine representations and include certain representations $\hat{\mathcal{P}}_{\langle m k, n\rangle}$ that are known as twisted highest weight modules. These are generated from a state $|m k, n\rangle$ satisfying the conditions

$$
\begin{aligned}
& \Psi_{r}^{ \pm}|m k, n\rangle=0 \text { for } r>\mp m \\
& E_{r}|m k, n\rangle=0=N_{r}|m k, n\rangle \text { for } r>0 \\
& E_{0}|m k, n\rangle=m k|m k, n\rangle, \quad N_{0}|m k, n\rangle=n|m k, n\rangle
\end{aligned}
$$

by applications of the generators $\Psi_{ \pm r}^{ \pm}, r \leq \mp m$, and $E_{r}, N_{r}, r>0$ (note the difference in (3.10) with the definition of ordinary highest weight modules which would involve $r>0$ instead). Let us note that for $m=0$, the construction gives us the Verma module of the projective cover $\mathcal{P}_{n}$. We also observe that in the twisted highest weight module, the eigenvalues of $L_{0}$ are bounded from below, simply because we can only apply a finite number of fermionic generators to descend from $|m k, n\rangle$. For $m \neq 0$ there are two states of lowest $L_{0}$ eigenvalue which are given by

$$
\prod_{r=1}^{|m|} \Psi_{r}^{-\operatorname{sign}(m)}|m k, n\rangle \quad, \quad \prod_{r=0}^{|m|} \Psi_{r}^{-\operatorname{sign}(m)}|m k, n\rangle .
$$


For $m \geq 0$ for instance, they possess $L_{0}$ eigenvalue $n m-m^{2} / 2-m / 2$ and transform in the typical representation $\langle m k, n-m\rangle$. Consequently, the module $\hat{\mathcal{P}}_{\langle m k, n\rangle}$ contains $\mathcal{V}_{\langle m k, n-m\rangle}$ as a subspace. If we divide by the latter we stay with a Verma module $\mathcal{V}_{\langle m k, n-m+1\rangle}$. This structure in encoded in the following diagram,

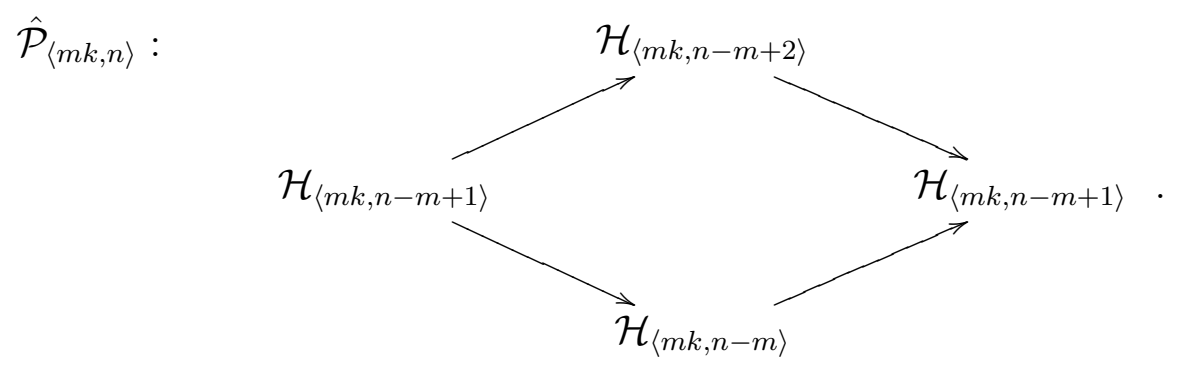

It is easy to see that the $\hat{\mathcal{P}}_{\langle m k, n\rangle}$ are the images of the $\mathcal{P}_{n}$ under the spectral flow. The picture does not only tell us how spectral flow acts on the various Verma modules we can built over representations with $E=0$, it also displays the twisted highest weight modules $\hat{P}$ as natural cousins of the projective covers $\mathcal{P}_{n}$.

\subsection{The state space of the GL(1|1) WZW model}

Having gained some insight into the representation theory of the gl(1|1) current algebra, it is very tempting to conjecture that the state space of the full field theory possesses exactly the same structure as the minisuperspace theory with only one extra feature: the theory is periodic under shifts of $e$ by multiples of the level $k$,

$$
\mathcal{H}_{\mathrm{WZW}}=\int_{e \neq m \bmod k} \operatorname{dedn} \mathcal{H}_{\langle-e,-n+1\rangle}^{L} \otimes \mathcal{H}_{\langle e, n\rangle}^{R} \oplus \sum_{m} \int_{0}^{1} d q \hat{\mathcal{J}}_{q}^{(m)} .
$$

Here $\hat{\mathcal{J}}_{q}, q \in[0,1[$, denotes a family of indecomposable representations of $\hat{g} l(1 \mid 1) \otimes \hat{\mathrm{g}} \mathrm{l}(1 \mid 1)$. They are built from irreducible representations according to the following diagram

$$
\begin{aligned}
& \mathcal{J}_{q}^{(m)}: \bigoplus_{a \in \mathbb{Z}} \mathcal{H}_{\langle m k, q+a\rangle} \otimes \mathcal{H}_{\langle-m k,-q-a\rangle} \\
& \longrightarrow \bigoplus_{a \in \mathbb{Z}} \mathcal{H}_{\langle m k, q+a+1\rangle} \otimes \mathcal{H}_{\langle-m k,-q-a\rangle} \oplus \bigoplus_{a \in \mathbb{Z}} \mathcal{H}_{\langle m k, q+a\rangle} \otimes \mathcal{H}_{\langle-m k,-q-a-1\rangle} \\
& \longrightarrow \bigoplus_{a \in \mathbb{Z}} \mathcal{H}_{\langle m k, q+a\rangle} \otimes \mathcal{H}_{\langle-m k,-q-a\rangle} .
\end{aligned}
$$

According to several remarks earlier on, it is clear that the Virasoro modes $L_{0}$ and $\bar{L}_{0}$ cannot be diagonalized on $\mathcal{H}_{\text {wzw }}$. As we recalled in the introduction, such a behavior 
is closely linked with the existence of logarithmic singularities in the operator product expansion of local fields in the model.

In the next three sections we shall prove that the state space of the WZW model does indeed possess the proposed form. First we shall set up a free field approach that will allow us to compute any correlation function in the system. Then we use this tool to calculate the 3-point couplings. Finally, we shall compute the 4-point functions and show that they factorize over the proposed set of states. Along the way we shall also prove that the theory possesses the conjectured spectral flow symmetry. Needless to add that we will also find the predicted logarithmic singularities in the correlation functions.

\section{Solutions of the full CFT}

Our aim now is to construct correlation function of the WZW model on the supergroup GL(1|1). We shall obtain our explicit formulas through a free field representation of the model.

\subsection{Free field theory approach}

We shall suggest here to think of the WZW model on the supergroup GL(1|1) as a perturbation of a free field theory. The latter is composed from a bosonic linear dilaton background and two chiral fermionic bc-systems with central charges $c_{ \pm}=-2$. Our split of the theory into this free field theory and an interaction does not preserve the chiral symmetries of the full model, but it is manifestly local. Hence, consistency of our correlation function is guaranteed but their relation with the GL(1|1) WZW model, and in particular the chiral symmetry, needs to be established.

To describe strings that move on the supergroup target GL(1|1) we would normally use the following WZW action

$$
S_{\mathrm{WZW}}=\frac{k}{4 \pi} \int_{\Sigma} d^{2} z\left(\partial X \bar{\partial} Y+\partial Y \bar{\partial} X-2 e^{-i Y} \bar{\partial} c_{-} \partial c_{+}\right) .
$$

Here, $X$ and $Y$ are bosonic field on the world-sheet and $c_{ \pm}$are fermionic. The invariant measure on the space of these fields is

$$
d \mu_{\mathrm{WZW}} \sim \mathcal{D} X \mathcal{D} Y \mathcal{D}\left(e^{\frac{-i}{2} Y} c_{-}\right) \mathcal{D}\left(e^{\frac{-i}{2} Y} c_{+}\right)
$$


We shall use another action $S$ to describe this system that contains an additional pair of chiral auxiliary fermions $b_{ \pm}$. It is built, starting from the following free field theory

$$
\begin{array}{r}
S_{0}=\frac{1}{4 \pi} \int_{\Sigma} d^{2} z(k \partial X \bar{\partial} Y+k \partial Y \bar{\partial} X-\partial Y \bar{\partial} Y+Q \mathcal{R} Y) \\
+\frac{1}{2 \pi} \int_{\Sigma} d^{2} z\left(b_{+} \partial c_{+}+b_{-} \bar{\partial} c_{-}\right) .
\end{array}
$$

Terms in the first line form a linear dilaton background whose charge $Q$ we fix to be $Q=i / 2$. Note that the linear dilaton term depends only on the $Y$ coordinate. Therefore the contribution $c^{b}=2$ of the bosonic fields to the central charge in independent of the background charge $Q$. In addition, the action $S_{0}$ contains two chiral bc-systems. We chose to have the chiral fields $c_{ \pm}$with conformal weight $\Delta_{ \pm}^{c}=0$ while their partners $b_{ \pm}$ are fields of weight $\Delta_{ \pm}^{b}=1$; the central charge is then $c_{ \pm}^{f}=-2$.

To the free field theory $S_{0}$ we now add an interaction term of the following simple form

$$
S\left(X, Y, b_{ \pm}, c_{ \pm}\right)=S_{0}+S_{\mathrm{int}}=S_{0}-\frac{1}{\pi k} \int_{\Sigma} d^{2} z e^{i Y} b_{-} b_{+} .
$$

Note that all $X$-independent fields have vanishing conformal weight. Hence, the interaction term is massless. In our treatment of the theory $S$ we work with the canonical measure

$$
d \mu \sim \mathcal{D} X \mathcal{D} Y \mathcal{D} c_{-} \mathcal{D} c_{+} \mathcal{D} b_{-} \mathcal{D} b_{+}
$$

On a formal level it is possible to compare the theory $S$ with the original WZW-model on the supergroup GL(1|1). The comparison makes use of a rather subtle relation between the involved measures

$$
d \mu_{\mathrm{WZW}} \mathcal{D}\left(b_{-}\right) \mathcal{D}\left(b_{+}\right) \sim \exp \left(\frac{1}{4 \pi} \int_{\Sigma} d^{2} z(-\partial Y \bar{\partial} Y+Q \mathcal{R} Y)\right) d \mu
$$

Once this is inserted into our theory $S$, we can integrate the auxiliary fields to recover the action of the WZW model.

We shall also need a free field representation for the vertex operators operators of our theory. Let us begin with the fields $V_{\langle e, n\rangle}$ that are associated to typical representations. We model them after the matrices $\varphi_{\langle e, n\rangle}$, i.e.

$$
V_{\langle e, n\rangle}(x, \bar{x})=: e^{i e X(x, \bar{x})+i(n-1) Y(x, \bar{x})}:\left(\begin{array}{cc}
1 & i c_{-}(x) \\
i e c_{+}(\bar{x}) & e c_{-}(x) c_{+}(\bar{x})
\end{array}\right)
$$


Their conformal dimension is given by

$$
\Delta_{(e, n)}=(2 n-3) \frac{e}{2 k}+\frac{e^{2}}{2 k^{2}} .
$$

Comparison with eq. (2.4) shows that we have dropped one term in the lower right corner of the matrix. When $e \neq 0$, the conformal dimension of the omitted vertex operator differs from the dimension of the other matrix elements so that in some sense (see below) we should consider the additional term as 'subleading'. Note that the vertex operators (4.19) may only be used for $e \neq 0$. We need a new prescription to deal with $e=0$. Once more, the expression may be motivated with the help of the matrix $\varphi_{\langle e, n\rangle}$ which, at $e=0$, degenerates to

$$
\varphi_{\langle 0, n\rangle}=\left(\begin{array}{cc}
e^{i(n-1) y} & i \eta_{-} e^{i(n-1) y} \\
0 & e^{i n y}
\end{array}\right) .
$$

Since the lower right corner contains only a single term, there is nothing we can omit and consequently the corresponding vertex operators are introduced by

$$
V_{\langle 0, n\rangle}(x, \bar{x})=\left(\begin{array}{cc}
: e^{i(n-1) Y(x, \bar{x})} & i c_{-}(x): e^{i(n-1) Y(x, \bar{x})}: \\
0 & : e^{i n Y(x, \bar{x})}:
\end{array}\right) .
$$

Recall that at $e=0$, there exists a second family of 2-dimensional representations $\langle\overline{0, n}\rangle$. The corresponding matrices $\bar{\varphi}_{\langle 0, n\rangle}$ of functions on the supergroup are given by

$$
\bar{\varphi}_{\langle 0, n\rangle}=\left(\begin{array}{cc}
e^{i n y} & 0 \\
i \eta_{+} e^{i(n-1) y} & e^{i(n-1) y}
\end{array}\right) .
$$

These may also be obtained from the matrices $\varphi_{\langle e, n\rangle}$, but we have to conjugate the latter with the matrix $W=e \sigma^{+}+\sigma^{-}$( $\sigma^{ \pm}$are Pauli matrices) before sending $e$ to zero. The associated vertex operators are constructed as

$$
\bar{V}_{\langle 0, n\rangle}(x, \bar{x})=\left(\begin{array}{cc}
: e^{i n Y(x, \bar{x})}: & 0 \\
i c_{+}(\bar{x}): e^{i(n-1) Y(x, \bar{x})}: & : e^{i(n-1) Y(x, \bar{x})}:
\end{array}\right) .
$$

Our matrices $\bar{\varphi}$ do contain the functions $e_{+}(0, n)$ which were not included in $\varphi$. Nevertheless, we are still missing all functions of the form

$$
\varphi_{n}=\eta_{-} \eta_{+} e^{i(n-1) y}
$$

The corresponding vertex operators are obtained in the obvious way through the formula

$$
V_{n}(x, \bar{x})=c_{-}(x) c_{+}(\bar{x}): e^{i(n-1) Y(x, \bar{x})}: .
$$


We shall think of the functions $\varphi_{n}$ and the vertex operators $V_{n}$ as being associated with 1-dimensional atypical representations of $\mathrm{gl}(1 \mid 1) .{ }^{4}$ Some readers might prefer to construct the additional series of objects with the help of projective covers, replacing our $\varphi_{n}$ through $4 \times 4$ matrices (the representation matrices of the supergroup elements). Similarly, vertex operators could then also be assembled into $4 \times 4$ matrices. At least in the case of the gl(1|1) model, such an alternative approach carries no more information than the one we have chosen here. Therefore, we shall continue to work with single component objects.

\subsection{Computation of correlation functions}

Let us denote the typical primary fields of the full interacting theory $S$ by $\Phi_{\langle e, n\rangle}$. Then our prescription for the m-point correlators of this theory is

$$
\left\langle\prod_{\nu=1}^{m} \Phi_{\left\langle e_{\nu}, n_{\nu}\right\rangle}\left(x_{\nu}, \bar{x}_{\nu}\right)\right\rangle=\sum_{s=0}^{\infty} \frac{1}{s !}\left\langle\left(\frac{-1}{\pi k} \int_{\Sigma} d^{2} z e^{i Y} b_{-} b_{+}\right)^{s} \prod_{\nu=1}^{m} V_{\left\langle e_{\nu}, n_{\nu}\right\rangle}\left(x_{\nu}, \bar{x}_{\nu}\right)\right\rangle_{0}
$$

We can employ the same formula if some of the $e_{\nu}$ vanish as long as we agree to insert the corresponding vertex operators $V_{\left\langle 0, n_{\nu}\right\rangle}$ for each such primary field into the correlators on the right hand side. Similarly, the prescription may be used to determine correlation functions involving the field theory analogue $\Phi_{n}$ of the functions $\varphi_{n}$ defined above.

The correlators on the right hand side are to be evaluated in the free field theory $S_{0}$. In order to determine the latter we shall use the following simple formula for correlators in the bosonic theory

$$
\begin{gathered}
\left\langle\prod_{\nu=1}^{m} V_{\left(e_{\nu}, n_{\nu}\right)}\left(x_{\nu}, \bar{x}_{\nu}\right)\right\rangle=\prod_{\nu<\mu}\left|x_{\nu}-x_{\mu}\right|^{-2 \alpha_{\nu \mu}} \delta\left(\sum_{\nu=1}^{m} n_{\nu}-n-1\right) \delta\left(\sum_{\nu=1}^{m} e_{\nu}\right) \\
\text { where } \alpha_{\nu \mu}=\left(1-n_{\nu}\right) \frac{e_{\mu}}{k}+\left(1-n_{\mu}\right) \frac{e_{\nu}}{k}-\frac{e_{\nu} e_{\mu}}{k^{2}} .
\end{gathered}
$$

Here, $V_{(e, n)}=: \exp (i e X+i(n-1) Y)$ : are standard bosonic vertex operators with a somewhat unusual shift in the labels. The charge conservation for the e-charge is standard. For the parameters $n_{\nu}$, the background charge $Q$ becomes relevant. The usual rules tell us that $\sum\left(n_{\nu}-1\right)=2 Q / i=1$.

In addition we will have to evaluate correlation functions in the chiral bc-systems. According to the usual rules, non-vanishing correlators on the sphere must satisfy \# $c_{ \pm}-$

\footnotetext{
${ }^{4}$ This does not mean that they transform under the atypical 1-dimensional representation. In fact, the action of $\operatorname{gl}(1 \mid 1)$ certainly mixes e.g. $\phi_{n}$ with components of $\phi_{\langle 0, m\rangle}$.
} 
$\# b_{ \pm}=1$, i.e. the number of insertions of $c_{ \pm}$must exceed the number of insertions of $b_{ \pm}$ by one. For such chiral correlation functions one obtains

$$
\left\langle\prod_{\nu=1}^{n} b_{-}\left(z_{\nu}\right) \prod_{\mu=1}^{n+1} c_{-}\left(x_{\mu}\right)\right\rangle_{0}=\frac{\prod_{\nu<\nu^{\prime}}\left(z_{\nu}-z_{\nu^{\prime}}\right) \prod_{\mu<\mu^{\prime}}\left(x_{\mu}-x_{\mu^{\prime}}\right)}{\prod_{\nu} \prod_{\mu}\left(z_{\nu}-x_{\mu}\right)}
$$

and a similar formula applies to $c_{+}$and $b_{+}$. This concludes our preparation. We can now turn to a calculation of the correlators.

It is quite instructive to compare the above expansion (4.20) for field theory correlators with our previous discussion of "correlation functions" in the minisuperspace limit. From charge conservation in the $Y$-direction (parameter $n$ ) we infer that the $s^{\text {th }}$ term in the expansion is nonzero if and only if

$$
s+\sum_{\nu=1}^{m}\left(n_{\nu}-1\right)=1
$$

Hence, the summation over $s$ in eq. (4.20) is equivalent to the summation over $p$ in our formula (2.6), the precise relation between the two summation parameters being $p=s+1$. In section 2.3 we saw that there could only be a finite number of terms. The same is true for the full field theory since the number of $b$-insertions has to be smaller that the number of possible $c$-insertions. Hence, terms with $s \geq m$ vanish so that the summation is finite. A slightly more detailed analysis shows once more that the term with $s=m-1$ vanishes as long as we only insert typical fields. Consequently, the last non-vanishing term appears at $p=s+1=m-1$, just as in the minisuperspace model. Let us anticipate, however, that correlators of the fields $\Phi_{n}$ do receive contributions from $s=m-1$.

\section{The 3-point functions of the GL(1|1) model}

For our evaluation of the 3-point functions we shall adopt the following strategy. To begin with, we shall construct the 3 -point functions of the typical fields $\Phi_{\langle e, n\rangle}$. In the limit where $e \rightarrow 0$, these include correlations involving fields $\Phi_{\langle 0, n\rangle}$ or $\bar{\Phi}_{\langle 0, n\rangle}$ so that we do not have to list the corresponding 3-point functions separately. All these correlators turn out to mimic very closely the minisuperspace theory, except from a minor but interesting quantum deformation. In a second step, we shall then also determine 3-point functions involving one or more insertions of the fields $\Phi_{n}$ that come with the functions $\varphi_{n}$. These correlation functions contain logarithms. 


\subsection{3 -point functions of typical fields}

The first important result of this subsection provides us with an explicit formula for the 3 -point correlator of typical field. It takes the form

$$
\begin{gathered}
\left\langle\Phi_{\left\langle e_{1}, n_{1}\right\rangle}(\infty) \Phi_{\left\langle e_{2}, n_{2}\right\rangle}(1) \Phi_{\left\langle e_{3}, n_{3}\right\rangle}(x)\right\rangle=\sum_{s=0,1} C_{s}\left(e_{1}, e_{2}, e_{3}\right) \frac{\left\langle\varphi_{\left\langle e_{1}, n_{1}\right\rangle} \varphi_{\left\langle e_{2}, n_{2}\right\rangle} \varphi_{\left\langle e_{3}, n_{3}\right\rangle}\right\rangle_{s}}{|1-x|^{2 \Delta_{s}}} \\
\text { where } \quad C_{0}\left(e_{1}, e_{2}, e_{3}\right)=1 \quad \text { and } \quad C_{1}\left(e_{1}, e_{2}, e_{3}\right)=\prod_{i=1}^{3} \Gamma\left(1+\frac{e_{i}}{k}\right) / \Gamma\left(1-\frac{e_{i}}{k}\right)
\end{gathered}
$$

The exponents in the denominator are given by $\Delta_{0}=\left(n_{2}-1\right) a_{3}+\left(n_{3}-1\right) a_{2}+a_{2} a_{3}$ and $\Delta_{1}=n_{2} a_{3}+n_{3} a_{2}+a_{2} a_{3}$ with the rescaled parameters $a_{i}=-e_{i} / k$. Note that for each choice of the parameters $n_{i}$ at most one of the two terms in the sum can contribute. Hence, our result is manifestly consistent with the conformal invariance of the model. The symbols $\langle f\rangle_{s}$ that appear in the numerator on the right hand side refer to the terms in the minisuperspace result for the 3-point function (see eq. (2.6), i.e.

$$
\begin{aligned}
\left\langle\varphi_{\left\langle e_{1}, n_{1}\right\rangle} \varphi_{\left\langle e_{2}, n_{2}\right\rangle} \varphi_{\left\langle e_{3}, n_{3}\right\rangle}\right\rangle= & \int d \mu \varphi_{\left\langle e_{1}, n_{1}\right\rangle} \varphi_{\left\langle e_{2}, n_{2}\right\rangle} \varphi_{\left\langle e_{3}, n_{3}\right\rangle}=\sum_{s=0,1}\left\langle\varphi_{\left\langle e_{1}, n_{1}\right\rangle} \varphi_{\left\langle e_{2}, n_{2}\right\rangle} \varphi_{\left\langle e_{3}, n_{3}\right\rangle}\right\rangle_{s} \\
\text { where } \quad & \left\langle\varphi_{\left\langle e_{1}, n_{1}\right\rangle} \varphi_{\left\langle e_{2}, n_{2}\right\rangle} \varphi_{\left\langle e_{3}, n_{3}\right\rangle}\right\rangle_{s}=G_{s}^{(3)} \delta\left(\sum_{\nu} e_{\nu}\right) \delta\left(\sum_{\nu} n_{\nu}-4+s\right)
\end{aligned}
$$

and $G_{s}^{(3)}$ are the unique invariant tensors in the triple tensor products of typical representations. Explicit formulas can be worked out by integration of the threefold products over the supergroup. We shall not need these formulas here. Since the terms in our result are proportional to the tensors $G_{s}^{(3)}$, our 3-point couplings are manifestly gl(1|1) covariant. The main difference between the minisuperspace limit and the full field theory result arises from the non-trivial, e-dependent factor $C_{1}$ in front of the second term. Note that the latter approaches $C_{1} \sim 1$ as we send $k$ to infinity, thereby reproducing the minisuperspace result in this limit.

Our formula for the 3-point functions is not very difficult to derive. Note that the first term with $s=0$ arises from the corresponding term in the expansion (4.20). Since there is no screening charge inserted in this case, the field theory computation is identical to the associated calculation in the minisuperspace theory and hence $C_{0}=1$. As for the second term, the computation is slightly more involved. Let us only compute one 
particular component here.

$$
\begin{gathered}
\left\langle{ }_{0}^{0} \Phi_{\left\langle e_{1}, n_{1}\right\rangle}(\infty){ }_{1}^{1} \Phi_{\left\langle e_{2}, n_{2}\right\rangle}(1){ }_{1}^{1} \Phi_{\left\langle e_{3}, n_{3}\right\rangle}(x)\right\rangle \sim \frac{1}{k} \int d^{2} z e_{2} e_{3} \frac{|z-1|^{2 a_{2}-2}|z-x|^{2 a_{3}-2}}{|1-x|^{2 \Delta_{0}-2}} \\
\quad=\frac{1}{k} \frac{e_{2} e_{3}}{|1-x|^{2 \Delta_{1}}} \frac{\Gamma\left(-a_{2}\right) \Gamma\left(1+a_{2}+a_{3}\right) \Gamma\left(-a_{3}\right)}{\Gamma\left(1+a_{3}\right) \Gamma\left(-a_{2}-a_{3}\right) \Gamma\left(1+a_{2}\right)}=C_{1}\left(e_{1}, e_{2}, e_{3}\right) \frac{e_{2}+e_{3}}{|1-x|^{2 \Delta_{1}}}
\end{gathered}
$$

The $\sim$ in the first line means that we only display the coefficient in front of the $\delta$-functions for the contribution with $s=1$ insertion of the interaction, i.e. we assume implicitly that $e_{1}=-e_{2}+e_{3}$ and $n_{1}=3-n_{2}-n_{3}$. In the computation we used a special case of the Dotsenko-Fateev integration formula (Appendix A). The other steps are straightforward. It is finally easy to see that $e_{2}+e_{3}$ arises as a result of the corresponding minisuperspace computation.

It is now rather instructive to study what happens to the component ${ }_{1}^{1} \Phi_{\langle e, n\rangle}$ as we send $e$ to zero. Since we are using the associated vertex operator $e c_{-} c_{+} V_{(e, n)}$ in the free field computation, one might naively expect that the limiting field is zero. Our formula for the 3-point coupling, however, shows that this is not the case. Instead we find

$$
\lim _{e \rightarrow 0}{ }_{1}^{1} \Phi_{\langle e, n\rangle}={ }_{0}^{0} \Phi_{\langle e, n+1\rangle}
$$

Though this result may appear a bit surprising at first, it is actually rather natural. In order to construct the vertex operator for typical fields, we had to remove one term from the corresponding matrix $\varphi_{\langle e, n\rangle}$ of the minisuperspace theory. We declared this term to be 'subleading' in some sense. But when $e$ is sent to zero, the 'leading term' in the lower right corner of $\varphi_{\langle e, n\rangle}$ vanishes so that the other term is no longer 'negligible'. This is exactly what we may infer from the previous formula.

Another remark concerns a very interesting new symmetry of the field theory that is not present in the minisuperspace theory. Note that the coefficients $C_{1}$ of the 3 -point couplings have poles whenever one of the $a_{i}$ becomes a positive integer. This behavior seems to distinguish the lines $e=k \mathbb{Z}$ in the parameter space of $\langle e, n\rangle$. In the minisuperspace theory, only the line $e=0$ was special. Hence, we take the behavior of $C_{1}$ as a first indication that the spectral flow symmetry might be a symmetry of our physical model, not just of its symmetry (see section 3). This is indeed the case. In fact, one may show by a short explicit computation that

$$
\left\langle\Phi_{\left\langle e_{1}, n_{1}\right\rangle}(\infty){ }_{0}^{0} \Phi_{\left\langle e_{2}, n_{2}\right\rangle}(1){ }_{1}^{1} \Phi_{\left\langle e_{3}, n_{3}\right\rangle}(0)\right\rangle=\mathcal{N}\left\langle\Phi_{\left\langle e_{1}, n_{1}\right\rangle}(\infty){ }_{1}^{1} \Phi_{\left\langle e_{2}+k, n_{2}-1\right\rangle}(1){ }_{0}^{0} \Phi_{\left\langle e_{3}-k, n_{3}+1\right\rangle}(0)\right\rangle
$$


for all the matrix components of $\Phi_{\left\langle e_{1}, n_{1}\right\rangle}$. The coefficient $\mathcal{N}=e_{3} /\left(e_{2}+k\right)$ is due to our normalization of the components ${ }_{1}^{1} \Phi$ and it would be absent had we normalized our fields in the canonical way. Consequently, the 3-point functions of typical fields are periodic in the parameter $e$ with period length $k$. This proves that spectral flow symmetry of the model on the level of its 3-point functions functions.

Let us finally recall that the 3-point correlators involving primaries $\Phi_{\langle 0, n\rangle}$ with $e=0$ may be obtained by taking $e_{i}$ to zero in the above expression of the 3-point couplings. In case of $\bar{\Phi}_{\langle 0, n\rangle}$ one should remember to conjugate the typical fields with the matrix $W=e \sigma^{+}+\sigma_{-}$before performing the limit. All the couplings in the resulting correlators agree with the minisuperspace result. In fact, when one of the labels $e_{i}=0$, the other two labels differ only by their sign and hence the coefficient $C_{1}=1$.

\subsection{3 -point functions involving $\Phi_{n}$}

It now remains to find correlation functions involving the fields $\Phi_{n} .{ }^{5}$ These can again be determined by explicit computation using our free field representation. Let us start by stating the result for a single insertion of the field $\Phi_{n}$,

$$
\begin{aligned}
\left\langle\Phi_{n_{1}}(\infty) \Phi_{\left\langle e_{2}, n_{2}\right\rangle}(1) \Phi_{\left\langle e_{3}, n_{3}\right\rangle}(x)\right\rangle=\frac{\left\langle\varphi_{n_{1}} \varphi_{\left\langle e_{2}, n_{2}\right\rangle} \varphi_{\left\langle e_{3}, n_{3}\right\rangle}\right\rangle}{|1-x|^{2 \Delta}} \\
+\frac{1}{k} \frac{\left\langle\varpi_{n_{1}} \varphi_{\left\langle e_{2}, n_{2}\right\rangle} \varphi_{\left\langle e_{3}, n_{3}\right\rangle}\right\rangle}{|1-x|^{2 \Delta}}\left(\mathcal{Z}+\vartheta\left(a_{2}\right)-\log |1-x|^{2}\right) \\
\text { where } \quad \vartheta(a)=2 \psi(1)-\psi(a)-\psi(1-a),
\end{aligned}
$$

the function $\varpi_{n}$ is defined as $\varpi_{n}=\exp ($ iny $)$, our symbol $\psi(a)=\Gamma^{\prime}(a) / \Gamma(a)$ denotes the Di-gamma function and the exponent $\Delta$ agrees with the exponents $\Delta_{0}=\Delta_{1}$ we introduced previously. Since a non-vanishing 3-point coupling requires $a_{2}=-a_{3}$ we obtain $\Delta=\left(n_{3}-n_{2}\right) a_{2}-a_{2}^{2}$. The constant $\mathcal{Z}$, finally, may be shifted through a field redefinition and therefore its exact value is irrelevant. In fact, if we substitute the atypical field $\Phi_{n}$ on the left hand side of the above equation by

$$
\tilde{\Phi}_{n}=\Phi_{n}+\kappa \cdot{ }_{0}^{0} \Phi_{\langle 0, n+1\rangle}
$$

then the 3-point function remains of the same form with $\mathcal{Z}$ being replaced by

$$
\tilde{\mathcal{Z}}=\mathcal{Z}+\kappa k \text {. }
$$

\footnotetext{
${ }^{5}$ Recall that the fields $\Phi_{n}$ and their minisuperspace counterparts $\varphi_{n}$ do not carry any matrix indices (see also our comments at the end of section 4.1).
} 
Let us note that with our original definition of the field $\Phi_{n}$, the constant $\mathcal{Z} \sim 1 /\left(e_{2}+e_{3}\right)$ turns our to be infinite.

It is not very difficult to prove the formula (5.24). With the help of our free field representation we find

$$
\begin{gathered}
\left\langle\Phi_{n_{1}}(\infty){ }_{1}^{1} \Phi_{\left\langle e_{2}, n_{2}\right\rangle}(1){ }_{0}^{0} \Phi_{\left\langle e_{3}, n_{3}\right\rangle}(x)\right\rangle \sim \frac{e_{2}}{k} \frac{\Gamma\left(a_{2}\right) \Gamma(-\epsilon) \Gamma\left(1-a_{2}+\epsilon\right)}{\Gamma\left(a_{2}-\epsilon\right) \Gamma\left(1-a_{2}\right) \Gamma(1+\epsilon)}|1-x|^{2 \epsilon} \\
=\frac{e_{2}}{k}\left(-\frac{1}{\epsilon}+2 \psi(1)-\psi\left(a_{2}\right)-\psi\left(1-a_{2}\right)-\log |1-x|^{2}+o(\epsilon)\right)
\end{gathered}
$$

Here we use the same conventions as in the previous subsection along with some results which may be found in the appendix. The expansion around $\epsilon=a_{2}+a_{3} \sim 0$ is performed using

$$
\Gamma(x+\epsilon) / \Gamma(x) \sim 1+\epsilon \psi(x)+o\left(\epsilon^{2}\right) .
$$

In the minisuperspace limit, the associated 3-point function assumes the value $e_{2}$. Hence, we have established eq. (5.24) with $\mathcal{Z} \sim-1 / \epsilon$, at least for one particular component.

Similar steps allow us to determine the correlator in case there are there two insertions of the field $\Phi_{n}$,

$$
\left\langle\Phi_{\left\langle e_{1}, n_{1}\right\rangle}(\infty) \Phi_{n_{2}}(1) \Phi_{n_{3}}(x)\right\rangle=\frac{2}{k}\left\langle\varphi_{\left\langle e_{1}, n_{1}\right\rangle} \varpi_{n_{2}} \varphi_{n_{3}}\right\rangle\left(\mathcal{Z}+\log |1-x|^{2}\right) .
$$

Here we use the same notation as in eq. (15.24). Note that a non-vanishing 3-point coupling with two insertions of $\Phi_{n}$ requires the third field to have $e_{1}=0$. In the minisuperspace correlator on the right hand side we could also have replaced $\varphi_{n_{3}}$ by $\varpi_{n_{3}}$ rather than performing this substitution on $\varphi_{n_{2}}$.

Finally, when all three fields are of the type $\Phi_{n}$, the 3-point couplings read

$$
\left\langle\Phi_{n_{1}}(\infty) \Phi_{n_{2}}(1) \Phi_{n_{3}}(x)\right\rangle=\frac{1}{k^{2}}\left\langle\varphi_{n_{1}} \varpi_{n_{2}} \varpi_{n_{3}}\right\rangle\left(3 \mathcal{Z}^{2}+2 \mathcal{Z} \log |1-x|^{2}-\left(\log |1-x|^{2}\right)^{2}\right) .
$$

The attentive reader might have noticed that the minisuperspace integral on the right hand side contains an infinite factor $\int d e=\delta(0)$. To understand such a behavior we recall that the fields we use are associated with $\delta$-function normalizable states. They can all be approached through a series of normalizable fields by smearing them with appropriate functions in $(e, n)$-space. Correlation functions are finite as long as one of the involved fields is normalizable. In our last correlator for three fields of the type $\Phi_{n}$, however, we had so set all the parameters $e$ to zero. Hence, from this 3-point function alone it is 
no longer possible to deduce any finite correlation functions in which at least one field would need to be normalizable (and hence to be smeared out in the $e$-coordinate). Having uncovered the rather trivial origin of the divergence, we shall no longer hesitate to write factors $\delta(0)$.

After this brief digression into mathematical subtleties it is instructive to compare our answers for the correlation functions of symplectic fermions (see e.g. 36]). The comparison shows that our typical vertex operators are very close relatives of the twist fields of the symplectic fermion while $\Phi_{n}$ behave like the logarithmic partner of the vacuum in that theory.

\section{The 4-point function and factorization}

We are finally in a position to show that the state space we have proposed at the end of the third section is consistent with the factorization of 4-point functions. To this end we shall now compute at least one special 4-point function of typical fields and we shall show that it factorizes over the conjectures set of possible intermediate states.

The 4-point function we are about to compute has the following form [24])

$$
G(x, \bar{x}):=\left\langle\Phi_{\left\langle-e^{\prime}+\varepsilon, 1-n^{\prime}\right\rangle}(\infty) \Phi_{\langle e, n\rangle}(x, \bar{x}) \Phi_{\langle-e-\varepsilon, 1-n\rangle}(1) \Phi_{\left\langle e^{\prime}, n^{\prime}\right\rangle}(0)\right\rangle .
$$

Let us note that the same 4-point function was also computed in 24] as a local solution of the corresponding Knizhnik-Zamolodchikov equation. Needless to say that our free field computations shall give the same answer. In any case, it is easy to see that this correlator only receives contributions from the insertion of one screening charge. In order to spell out the result, we rely heavily on the notations that are introduced throughout this work (in particular in subsection 2.3 and in appendix A). Furthermore, it will be convenient to work with the rescaled parameters

$$
a=-\frac{e}{k} \quad, \quad a^{\prime}=-\frac{e^{\prime}}{k} \quad, \quad \alpha=-\frac{\varepsilon}{k} .
$$

With these notations, the four point function $G$ can be written in the following form

$$
\begin{gathered}
G(x, \bar{x})=\frac{1}{2 k}|x|^{2 \beta}|1-x|^{2 \gamma} \sum_{\sigma_{i}, \bar{\sigma}_{i}}^{\prime} E_{\bar{\sigma}_{1} \bar{\sigma}_{2} \bar{\sigma}_{3} \bar{\sigma}_{4}}^{\sigma_{1} \sigma_{2} \sigma_{3} \sigma_{4}} \mathcal{G}(x, \bar{x})_{\sigma_{1} \sigma_{2} \sigma_{3} \sigma_{4}}^{\bar{\sigma}_{1} \bar{\sigma}_{2} \bar{\sigma}_{3} \bar{\sigma}_{4}} \\
\text { where } \\
\qquad \begin{array}{c}
\beta=(1-n) a^{\prime}+\left(1-n^{\prime}\right) a+a a^{\prime} \\
\gamma=(n-1)(\alpha+a)+n a+a(\alpha+a)
\end{array}
\end{gathered}
$$


where the summation extends over all spin configurations such that $\sum_{i} \sigma_{i}=\sum_{i} \bar{\sigma}_{i}=2$. Note that this includes complementary spin assignments, i.e. configurations satisfying $\sigma_{i}+\bar{\sigma}_{i}=1$ for all $i$, which can not arise in the minisuperspace theory. It remains to spell out the functions $\mathcal{G}$. Up to a sign, they are given by

$$
\begin{aligned}
& \mathcal{G}(x, \bar{x})=\left(-e^{\prime}+\varepsilon\right)^{\bar{\sigma}_{1}} e^{\bar{\sigma}_{2}}(-e-\varepsilon)^{\bar{\sigma}_{3}}\left(e^{\prime}\right)^{\bar{\sigma}_{4}} x^{\sigma_{2} \sigma_{4}}(x-1)^{\sigma_{2} \sigma_{3}} \bar{x}^{\bar{\sigma}_{2} \bar{\sigma}_{4}}(\bar{x}-1)^{\bar{\sigma}_{2} \bar{\sigma}_{3}} \times \\
& \quad \times(-1)^{\sum_{\nu \leq \mu \leq 3} \bar{\sigma}_{\nu} \sigma_{\mu}}\left[\left|\mathcal{F}\left(a+\sigma_{2}, a^{\prime}-\alpha+\sigma_{2}+\sigma_{3}+\sigma_{4}-1 ; a+a^{\prime}+\sigma_{2}+\sigma_{4} \mid x\right)\right|^{2}+\right. \\
& \left.+(-1)^{\sigma_{2}+\sigma_{3}+\bar{\sigma}_{2}+\bar{\sigma}_{3}}\left|x^{1-\sigma_{2}-\sigma_{4}-a-a^{\prime}} \mathcal{F}\left(\sigma_{3}-\alpha-a, 1-\sigma_{4}-a^{\prime} ; 2-\sigma_{2}-\sigma_{4}-a-a^{\prime} \mid x\right)\right|^{2}\right]
\end{aligned}
$$

Here, the notation $|.|^{2}$ means that we we multiply the argument with an identical factor in which $\mathcal{F}, \sigma_{i}$ and $x$ have been replaced by the bared quantities. In this form, it is possible to compare our result with the expressions that were found in [24].

We are now interested in the closed string states that propagate in the intermediate channel when $\alpha \rightarrow 0$. It is convenient to rewrite the function $\mathcal{G}$ at $\alpha=0$ in terms of the variables $m=1-\sigma_{2}-\sigma_{3}$ and $\bar{m}=1-\bar{\sigma}_{2}-\bar{\sigma}_{3}$ which may assume values $m, \bar{m}=-1,0,1$,

$$
\begin{aligned}
\mathcal{G}(x, \bar{x}) & =(-1)^{\bar{\sigma}_{1}+\bar{\sigma}_{3}}\left(e^{\prime}\right)^{\bar{\sigma}_{1}+\bar{\sigma}_{4}} e^{\bar{\sigma}_{2}+\bar{\sigma}_{3}}(x-1)^{\sigma_{2} \sigma_{3}}(\bar{x}-1)^{\bar{\sigma}_{2} \bar{\sigma}_{3}}(-1)^{\sum_{\nu \leq \mu \leq 3} \bar{\sigma}_{\nu} \sigma_{\mu}} \times \\
& \times\left[\left|\mathcal{F}\left(a+\sigma_{2}, a^{\prime}+\sigma_{2}+\sigma_{3}+\sigma_{4}-1 ; a+a^{\prime}+2 \sigma_{2}+\sigma_{3}+\sigma_{4}-1+m \mid x\right)\right|^{2}+\right. \\
+ & \left.(-1)^{m+\bar{m}}\left|x^{1-\sigma_{2}-\sigma_{4}-a-a^{\prime}} \mathcal{F}\left(\sigma_{3}-a, 1-\sigma_{4}-a^{\prime} ; 1+\sigma_{3}-\sigma_{4}-a-a^{\prime}+m \mid x\right)\right|^{2}\right]
\end{aligned}
$$

Since the third argument of the function $\mathcal{F}$ coincides with the sum of the first two arguments up to an integer, we expect logarithms to appear when we expand the correlators $G$ around $x=1$,

$$
\begin{gathered}
G(x, \bar{x}) \stackrel{x \rightarrow 1}{\sim} \frac{1}{|1-x|^{2 \Delta}}\left(\left\langle\varphi_{\left\langle-e^{\prime}+\varepsilon, 1-n^{\prime}\right\rangle} \varphi_{\langle e, n\rangle} \varphi_{\langle-e-\varepsilon, 1-n\rangle} \varphi_{\left\langle e^{\prime}, n^{\prime}\right\rangle}\right\rangle\right. \\
\left.\quad+\frac{e e^{\prime}}{k} \delta_{m, 0} \delta_{\bar{m}=0}\left(\log |1-x|^{2}-\vartheta(a)-\vartheta\left(a^{\prime}\right)\right)\right)+\ldots
\end{gathered}
$$

where the exponent $\Delta=-\gamma(\alpha=0)$ is given by $\Delta=(1-2 n) a-a^{2}$. The function $\vartheta$ was introduced in eq. (5.25).

We would finally like to show that formula (6.28) is consistent with the factorization through the set of proposed states. The proof employs the following expression for the 
operator product expansion

$$
\begin{aligned}
& \Phi_{\langle e, n\rangle}(x) \Phi_{\langle-e, 1-n\rangle}(1) \sim \frac{1}{|1-x|^{2 \Delta}}\left(\int d l\left\langle\varpi_{l} \varphi_{\langle e, n\rangle} \varphi_{\langle-e, 1-n\rangle}\right\rangle \Phi_{l}(1)+\right. \\
& \left.+\int d l\left\langle\varphi_{l} \varphi_{\langle e, n\rangle} \varphi_{\langle-e, 1-n\rangle}\right\rangle \Omega_{l}(1)+\frac{1}{k}\left\langle\varpi_{l} \varphi_{\langle e, n\rangle} \varphi_{\langle-e, 1-n\rangle}\right\rangle\left(\vartheta(a)-\mathcal{Z}-\log |1-x|^{2}\right) \Omega_{l}(1)\right) . .
\end{aligned}
$$

where $\Omega_{l}$ is a shorthand for $\Omega_{l}={ }_{0}^{0} \Phi_{\langle 0, l+1\rangle}$. This operator product is a direct consequence of our results on 3 -point functions. In order to verify the sign in front of the constant $\mathcal{Z}$ on the right hand side one uses that

$$
\left\langle\Phi_{n}(\infty) \Phi_{n^{\prime}}(1)\right\rangle=2 \mathcal{Z} \delta(0) \delta\left(n+n^{\prime}-2\right)
$$

After inserting this operator product into the 4-point correlator, we can evaluate the resulting terms with the help of eq. (5.24). The first terms in each line of eq. (6.29) obviously combine into the first term of formula (6.28). We may evaluate the contribution from the last term on the right hand side of eq. (6.29) with the help of

$$
\left\langle\varpi_{l} \varphi_{\langle e, n\rangle} \varphi_{\langle-e, 1-n\rangle}\right\rangle=-e \delta_{m, 0} \delta_{\bar{m}, 0} \delta(l-1)
$$

As before, the formula should be read as a set of equations for the matrix components

${ }_{\bar{\sigma}}^{\sigma} \varphi_{\langle e, n\rangle}$ and ${\overline{\sigma^{\prime}}}^{\sigma^{\prime}} \varphi_{\langle e, n\rangle}$. The quantities $m$ and $\bar{m}$ on the right hand side are defined through $m=1-\sigma-\sigma^{\prime}$ and $\bar{m}=1-\bar{\sigma}-\bar{\sigma}^{\prime}$. Putting all this together we arrive at the second term in formula (6.28). Consequently, we have confirmed that our 4-point functions factorize on the set of states we had predicted.

\section{Concluding remarks}

In this note we have constructed the correlators of the GL(1|1) WZW model through a free field representation and we have investigated some properties of the theory. We have seen in particular that some correlators of the model contain logarithmic singularities. Let us stress once more that special 4-point functions of this theory had been computed before 24. Rozansky and Saleur had also observed the logarithms which appear whenever the intermediate states are associated with atypical representations. The new aspect of our approach here is that we were able to relate this very closely to the geometry (harmonic analysis) of supergroups. To the best of our knowledge, this is the first time that a family 
of logarithmic conformal field theories comes with a geometric interpretation. This may well prove to be a valuable source for further insights.

While our formulas for the 4-point functions agree with those of [24, it is not clear how other aspects relate in detail. We notice in particular that in 24], the need to build non trivial knot invariants led to special regularizations. These include e.g. a prescription to eliminate the divergence in the $\mathcal{Z}$ factors above. In addition, the characters of two dimensional representations were required to be orthogonal, even though their natural scalar product is always zero - as a result, the metric used in [35] (formula 148 of that paper) differs by a factor $e$ from the invariant metric. In any case, it is very possible that, for particular values of $k$ (especially in the strong quantum regime), other consistent quantum theories appear.

Another comment concerns an argument in [37] which suggest that the GL(1|1) model is a rather trivial example of a logarithmic conformal field theory. This assessment is based on the observation that its stress energy tensor

$$
T=\frac{1}{2 k}\left(2 N E+\Psi^{-} \Psi^{+}-\Psi^{+} \Psi^{-}\right)+\frac{1}{2 k^{2}} E^{2}
$$

is the bottom component of a projective representation under the right current algebra, with the top component being

$$
t=\frac{1}{8 k^{2}}\left(2 N E-\Psi^{-} \Psi^{+}+\Psi^{+} \Psi^{-}\right)+\frac{1}{2 k} N^{2}
$$

and two intermediate fermionic components of the form

$$
\begin{gathered}
\left\{\Psi^{-}, t\right\}=\frac{1}{2 k^{2}} E \Psi^{-}+\frac{1}{2 k}\left(N \Psi^{-}+\Psi^{-} N\right) \\
\left\{\Psi^{+}, t\right\}=-\frac{1}{2 k^{2}} E \Psi^{+}-\frac{1}{2 k}\left(N \Psi^{+}+\Psi^{+} N\right)
\end{gathered}
$$

The operator product expansion between the bosonic components $t$ and $T$,

$$
T(z) t(w)=\frac{1}{2 k} \frac{1}{(z-w)^{4}}+\frac{2 t(w)}{(z-w)^{2}}+\frac{\partial t}{z-w}
$$

can be used to argue very easily that $L_{0}$ is diagonalizable on this multiplet, a conclusion which is not in contradiction with anything we have said before since the identification between $L_{0}$ and the Casimir element applies only to highest weight states. Looking at this one multiplet alone, it would seem that more "interesting" logarithmic theories [37] are 
those for which the Virasoro field $T$ appears together with its partner $t$ on the right hand side of the operator product (17.33). In fact, the action of $L_{0}$ in the Virasoro multiplet of such theories ceases to be diagonalizable Let us stress, however, that the GL(1|1) WZW model is much richer than this observation would suggest. As our results show, it possesses many multiplets with non-diagonalizable $L_{0}$ e.g. even within the space of ground states. The top component of another potentially interesting multiplet can be obtained with $L_{-2}$ on $\Phi_{1}$.

Even though our analysis here was carried out for GL(1|1), we do not expect the results to be much different for compactified $\mathrm{U}(1 \mid 1)$ model. In the latter case, the spectrum of $e$ and $n$ should be discrete, and, in the full quantum field theory, winding will have to be introduced.

Irrespective of whether we choose $\mathrm{U}(1 \mid 1)$ or $\mathrm{GL}(1 \mid 1)$, we note that the spectrum of the theory is not bounded from below. This is expected since the $\mathrm{gl}(1 \mid 1)$ metric is not positive definite - a fact manifest, for instance, in that the naive functional integral for the free field representation say (4.14) is divergent. This feature is generic of supergroups, and it was suggested by Zirnbauer in particular [4] that the WZW model could only be defined by trading the target space for a Riemannian symmetric superspace with real submanifold $H^{1} \times S^{1}$. We have not followed that route here, observing instead that quantum mechanics on GL(1|1) was well defined, and assuming that there existed a quantum field theory reducing to it in the minisuperspace limit.

Let us finally point out that the geometric arguments that lead to the existence of indecomposables in the spectrum were not specific to the particular model under consideration. All they required was the presence of a Lie-superalgebra symmetry and the existence of the identity field in the spectrum of the theory. The latter always sits in an atypical representation and is - at least whenever the theory contains a typical field multiplet - part of a larger indecomposable projective representation. The existence of an identity field also has a rather simple geometric origin: it appears for all theories in which the bosonic manifold of the target space is compact. For non-compact target spaces, the identity can only be part of the spectrum if it may be approximated by normalizable functions. This is the case for flat target spaces, i.e. in the example we have studied. In more generic non-compact curved backgrounds, however, the identity is separated by a gap from the normalizable states of the theory. We therefore conclude that models with a compact (or flat) target space and a Lie superalgebra symmetry provide examples of 
logarithmic conformal field theory. This is certainly a vast class.

The insights of this note might be relevant also for non-compact backgrounds once we admit world-sheets with boundaries. In geometric terms, the boundary conditions we impose along the various boundary components are interpreted as branes. Such branes wrap certain subsets of the target space which may be either non-compact or compact. In the latter case, the boundary spectrum does contain an identity field even if the bulk spectrum does not. For branes that preserve some Lie superalgebra symmetries we are therefore back with a setup that resembles the one we discussed in the previous paragraph. Therefore we expect to find logarithmic singularities in the boundary correlators of a compact brane theory. We plan to come back to such issues in a forthcoming publication.

Acknowledgment: We wish to thank Anne Taormina for her participation in the early stages of this project and Thomas Quella for numerous comments for pointing out several mistakes in earlier versions of this text. We also acknowledge useful discussions/exchange with Michael Flohr, Matthias Gaberdiel, Gerhard Götz, Andreas Ludwig, Nick Read, Sylvain Ribault and Didina Serban. VS would like to thank the organizers of the string theory programmes at the Fields Institute, Toronto, and the KITP in Santa Barbara for their hospitality during the final stage of the work. This research was partially supported by the EU Research Training Network grants "Euclid", contract number HPRN-CT2002-00325, "ForcesUniverse", contract number MRTN-CT-2004-005104, by the National Science Foundation under Grant No. PHY99-0794, and by the Department of Energy.

\section{Appendix A: Some integral formulas}

Correlation functions can be computed from the free field representation using the following simple consequence of the Dotsenko-Fateev integral formula,

$$
\begin{aligned}
& \frac{1}{\pi} \int d^{2} z z^{a} \bar{z}^{\bar{a}}(1-z)^{b}(1-\bar{z})^{\bar{b}}(z-x)^{c}(\bar{z}-\bar{x})^{\bar{c}} \\
& \quad=|\mathcal{F}(-c,-c-1-a-b ;-c-a \mid x)|^{2}+(-1)^{c-\bar{c}+b-\bar{b}}\left|x^{a+c+1} \mathcal{F}(-b, a+1 ; a+c+2 \mid x)\right|^{2}
\end{aligned}
$$


We expressed the result of the integration through the following functions

$$
\begin{aligned}
\mathcal{F}(a, b ; c \mid x) & =\frac{\Gamma(c-b) \Gamma(b)}{\Gamma(c)}{ }_{2} F_{1}(a, b ; c \mid x) \\
\overline{\mathcal{F}}(\bar{a}, \bar{b} ; \bar{c} \mid \bar{x}) & =\frac{\Gamma(1-\bar{c})}{\Gamma(1-\bar{c}+\bar{b}) \Gamma(1-\bar{b})}{ }_{2} F_{1}(\bar{a}, \bar{b} ; \bar{c} \mid \bar{x}) .
\end{aligned}
$$

Validity of the integration formula requires that all the differences $a-\bar{a}, b-\bar{b}$ and $c-\bar{c}$

are integers. When one pair of exponents, e.g. the labels $a, \bar{a}$, vanishes, then the result simplifies to

$$
\frac{1}{\pi} \int d^{2} z(1-z)^{b}(1-\bar{z})^{\bar{b}}(z-x)^{c}(\bar{z}-\bar{x})^{\bar{c}}=|\mathcal{F}(-c,-c-1-b ;-c \mid x)|^{2} .
$$

This integral formula is used frequently in our evaluation of the 3-point couplings. For generic values of $b, c$ we have

$$
|\mathcal{F}(-c,-c-1-b ;-c \mid x)|^{2}=\frac{\Gamma(1+b) \Gamma(-1-c-b) \Gamma(1+\bar{c})}{\Gamma(-c) \Gamma(-\bar{b}) \Gamma(2+\bar{c}+\bar{b})}|1-x|^{2+b+c+\bar{b}+\bar{c}} .
$$

\section{References}

[1] G. Parisi and N. Sourlas, Self avoiding walk and supersymmetry, J. Phy. Lett. (Paris) 41 L403 (1980).

[2] K. B. Efetov, Supersymmetry And Theory Of Disordered Metals, Adv. Phys. 32, 53 (1983).

[3] H. A. Weidenmüller and M. Zirnbauer, Instanton approximation to the graded non linear sigma model for the integer quantum Hall effect, Nucl. Phys. B 305, 339 (1988).

[4] M. R. Zirnbauer, Conformal field theory of the integer quantum Hall plateau transition, arXiv hep-th/9905054.

[5] N. Read and H. Saleur, Exact spectra of conformal supersymmetric nonlinear sigma models in two dimensions, Nucl. Phys. B 613, 409 (2001) [arXiv:hep-th/0106124.

[6] S. Sethi, Supermanifolds, rigid manifolds and mirror symmetry, Nucl. Phys. B 430 (1994) 31 [arXiv:hep-th/9404186. 
[7] A. Schwarz, Sigma models having supermanifolds as target spaces, Lett. Math. Phys. 38 (1996) 91 [arXiv:hep-th/9506070].

[8] M. Aganagic and C. Vafa, Mirror symmetry and supermanifolds, arXiv:hep-th/0403192.

[9] A. Kapustin, Topological strings on noncommutative manifolds, Int. J. Geom. Meth. Mod. Phys. 1 (2004) 49 [arXiv:hep-th/0310057].

[10] V. Mathai and J. M. Rosenberg, On mysteriously missing T-duals, H-flux and the T-duality group, arXiv hep-th/0409073.

[11] V. Mathai and J. M. Rosenberg, T-duality for torus bundles via noncommutative topology, Commun. Math. Phys. 253 (2004) 705 [arXiv/hep-th/0401168.

[12] R. R. Metsaev and A. A. Tseytlin, Type IIB superstring action in AdS(5) x S(5) background, Nucl. Phys. B 533 (1998) 109 [arXiv hep-th/9805028].

[13] N. Berkovits, C. Vafa and E. Witten, Conformal field theory of AdS background with Ramond-Ramond flux, JHEP 9903 (1999) 018 [arXiv hep-th/9902098].

[14] M. Bershadsky, S. Zhukov and A. Vaintrob, $P S L(n \mid n)$ sigma model as a conformal field theory, Nucl. Phys. B 559 (1999) 205 [arXiv hep-th/9902180].

[15] J. de Boer and S. L. Shatashvili, Two-dimensional conformal field theories on $A d S(2 d+1)$ backgrounds, JHEP 9906, 013 (1999) [arXiv hep-th/9905032.

[16] N. Berkovits, M. Bershadsky, T. Hauer, S. Zhukov and B. Zwiebach, Superstring theory on AdS(2) x S(2) as a coset supermanifold, Nucl. Phys. B 567, 61 (2000) [arXiv hep-th/9907200].

[17] I. Bena, J. Polchinski and R. Roiban, Hidden symmetries of the AdS(5) $x S^{* * 5}$ superstring, Phys. Rev. D 69, 046002 (2004) [arXiv:hep-th/0305116].

[18] N. Mann and J. Polchinski, Bethe ansatz for a quantum supercoset sigma model, arXiv hep-th/0508232.

[19] S. Guruswamy, A. LeClair and A. W. W. Ludwig, gl(N|N) super-current algebras for disordered Dirac fermions in two dimensions, Nucl. Phys. B 583, 475 (2000) [arXiv cond-mat/9909143]. 
[20] M. J. Bhaseen, I. I. Kogan, O. A. Solovev, N. Tanigichi and A. M. Tsvelik, Towards a Field Theory of the Plateau Transitions in the Integer Quantum Hall Effect, Nucl. Phys. B 580, 688 (2000) [arXiv: cond-mat/9912060.

[21] H. Saleur and B. Wehefritz-Kaufmann, Integrable quantum field theories with OSP(m/2n) symmetries, Nucl. Phys. B 628 (2002) 407 [arXiv hep-th/0112095].

[22] F. H. L. Essler, H. Frahm and H. Saleur, Continuum Limit of the Integrable sl(2|1) $3-\overline{3}$ Superspin Chain, Nucl. Phys. B 712, 513 (2005) [arXiv cond-mat/0501197.

[23] A. M. Semikhatov, A. Taormina and I. Yu Tipunin, Higher level Appell functions, modular transformations and characters [arXiv:math.QA/0311314.

[24] L. Rozansky and H. Saleur, Quantum field theory for the multivariable AlexanderConway polynomial, Nucl. Phys. B 376 (1992) 461.

[25] V. Gurarie, Logarithmic operators in conformal field theory, Nucl. Phys. B 410, 535 (1993) [arXiv:hep-th/9303160.

[26] M. R. Gaberdiel and H. G. Kausch, A local logarithmic conformal field theory, Nucl. Phys. B 538, 631 (1999) [arXiv hep-th/9807091.

[27] J. S. Caux, I. I. Kogan and A. M. Tsvelik, Logarithmic operators and hidden continuous symmetry in critical disordered models, Nucl. Phys. B 466 (1996) 444 [arXiv:hep-th/9511134.

[28] Z. Maassarani and D. Serban, Non-unitary conformal field theory and logarithmic operators for disordered systems, Nucl. Phys. B 489 (1997) 603 [arXiv hep-th/9605062.

[29] V. Gurarie and A. W. W. Ludwig, Conformal field theory at central charge $c=0$ and two-dimensional critical systems with quenched disorder, [arXiv:hep-th/0409105.

[30] M. R. Gaberdiel, An algebraic approach to logarithmic conformal field theory, Int. J. Mod. Phys. A 18, 4593 (2003) [arXiv:hep-th/0111260.

[31] M. Flohr, Bits and pieces in logarithmic conformal field theory, Int. J. Mod. Phys. A 18 (2003) 4497 [arXiv:hep-th/0111228. 
[32] J. Teschner, The mini-superspace limit of the $S L(2, C) / S U(2)$ WZNW model, Nucl. Phys. B 546 (1999) 369 [arXiv hep-th/9712258.

[33] A. Huffmann, On representations of supercoalgebras, J. Phys. A 27, 6421 (1994) [arXiv:hep-th/9403100.

[34] G. Gotz, T. Quella and V. Schomerus, Representation theory of sl(2|1), arXiv:hep-th/0504234.

[35] L. Rozansky and H. Saleur, $S$ and T matrices for the superU(1,1) WZW model: Application to surgery and three manifolds invariants based on the Alexander-Conway polynomial, Nucl. Phys. B 389 (1993) 365 [arXiv:hep-th/9203069].

[36] H. G. Kausch, Symplectic fermions, Nucl. Phys. B 583, 513 (2000) [arXiv:hep-th/0003029.

[37] V. Gurarie, c-Theorem for disordered systems, Nucl. Phys. B 546, 765 (1999). 